Pacific Journal of Mathematics

DIFFERENCE ALGEBRAS OF LINEAR TRANSFORMATIONS 


\title{
DIFFERENCE ALGEBRAS OF LINEAR TRANSFORMATIONS ON A BANACH SPACE
}

\author{
BERTRAM YOOD
}

1. Introduction. Let $\Leftarrow(X)$ be the Banach algebra of all bounded linear transformations defined on an infinite-dimensional Banach space $\chi$ and with range in $X$. Let $\Re(X)$ be the set of completely continuous transformations contained in $\mathscr{S}(X)$. It is well knnwn that $\mathscr{f}(\mathscr{X})$ is a closed two-sided ideal in $(\subseteq(X)$. Thus, under the usual definitions, the difference algebra $(S(X)-\mathscr{f}(X)$ is again a Banach algebra. Let $\pi$ be the canonical homomorphism of $(\mathscr{E}(X)$ onto $(\mathcal{S}(X)-\mathfrak{A}(X)$.

The algebraic nature of $(F(X)-\mathscr{X}(\mathfrak{X})$ differs from that of $(\mathscr{E}(\mathfrak{X})$. In particular $(\mathscr{F}(X)$ is semi-simple while $\mathscr{E}(X)-\mathscr{I}(X)$ need not be semi-simple. An example of this is provided by taking for $\nVdash$ the Banach space $L(S)$ of Lebesgue-integrable numerical functions defined on, say, the unit interval $S$. If $T$ and $U$ are in $(\mathscr{X}(X)$ and are weakly completely continuous then $T U$ is completely continuous as shown by Dunford and Pettis [5, p. 370]. From this it follows readily that the image of the set of weakly completely continuous transformations in $(\xi(X)$ under $\pi$ is contained in the radical $\xi_{1}$ of $\mathscr{S}(X)-\mathscr{X}(X)$. Hence $(\mathscr{X}(X)-\Re(X)$

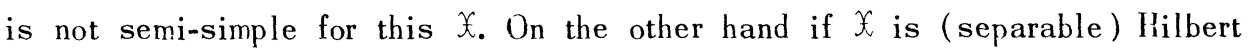
space, then $\mathscr{E}(X)-\mathscr{I}(X)$ is semi-simple.

In this paper we begin an investigation of the algebra $(\mathcal{E}(X)-\mathscr{A}(X)$. In particular its radical and its set of regular elements are examined. This turns out to be useful in the study of certain properties of transformations in $(E,(X)$.

In $\$ 3$ the inverse image $\pi^{-1}\left(\Re_{1}\right)$ of the radical is characterized. One formulation for this is that $\pi^{-1}\left(\Re_{1}\right)$ is the set of all $U \in(\mathcal{S}(X)$ such that $(T+U)(X)$ is closed and $(T+U)^{-1}(0)$ is finite-dimensional for all $T$ which are regular in $(f(X)$.

A well-known result of Schauder [13] asserts that if $I$ is the identity in $\mathbb{E}(X)$, and $U \in \Re(X)$, then $I+U$ and its adjoint $I^{*}+U^{*}$ have the same (finite) nullity. In $\S 4$ we obtain a generalization of this result as a reflection of the internal structure of $\left(\mathscr{E}(X)-\pi^{-1}\left(\Re_{1}\right)\right.$. Let $\Subset$ be any subset of $\mathscr{E}(\mathfrak{X})$ containing

Received May 7, 1953. Presented to the American Mathematical Society, February 23, 1952.

Pacific J. Math. 4 (1954), 615-636 
I such that $(1) \pi(\mathbb{E})$ is a multiplicative group and (2) the closure of the component of $E$ containing $I$ intersects $\pi^{-1}\left(\Re_{1}\right)$. Then there is a subring $E_{1}$ of $\mathscr{E}(\mathfrak{X})$ where the images of $E$ and $\mathscr{E}_{1}$ are geometrically related in $E(X)-\pi^{-1}\left(\Re_{\Re_{1}}\right)$ such that (a) $\widetilde{\Xi}_{1} \supset \pi^{-1}\left(\Re_{1}\right),\left(\right.$ b) $\pi\left(\widetilde{S}_{1}\right)$ is a group under the circle operation (see $\S 4$ ) where for each $T \in \widetilde{S}, U \in \widetilde{S}_{1}$ the quantities nul $T$, nul $T^{*}$, and nul $(T+U)$, nul $\left(T^{*}+U^{*}\right)$ are all finite and

$$
\operatorname{nul}\left(T^{*}+U^{*}\right)-\operatorname{nul}(T+U)=\operatorname{nul}\left(T^{*}\right)-\operatorname{nul}(T) .
$$

For $\subseteq$ the set of nonzero scalar multiples of $I$ this result already improves Schauder's, for there

$$
\widetilde{S}_{1}=\pi^{-1}\left(\eta_{1}\right)
$$

and since

$$
\operatorname{nul}(I)=\operatorname{nul}\left(I^{*}\right)=0
$$

we have

$$
\operatorname{nul}\left(I^{*}+U^{*}\right)=\operatorname{nul}(I+U)
$$

for every $U \in \pi^{-1}\left(\Re_{1}\right)$.

Let

$$
f(T)=\operatorname{nul}\left(T^{*}\right)-\operatorname{nul}(T) .
$$

This is known $[1,15]$ to be defined (finite) for the inverse image under $\pi$ of the set of regular elements of $\mathscr{E}(\mathfrak{X})-\mathfrak{I}(\mathfrak{X})$. Atkinson [1] has shown that the equation $f(T U)=f(T)+f(U)$ is satisfied. In $\S 5$ this is obtained as an application of the theory of functionals on an abstract semi-group. These considerations lead in $\S 6$ to a detailed study of the relation of the sets in $\mathscr{E}(\mathfrak{X})$ of elements with a one-sided or two-sided inverse to the corresponding sets, in $\mathbb{E}(\mathfrak{X})-\mathfrak{I}(\mathfrak{X})$.

2. Notation and preliminaries. Let $\mathfrak{X}$ be an infinite-dimensional Banach space and let $\mathscr{E}(\mathfrak{X})$ be the algebra of all bounded linear transformations defined on $X$ into $X$ made into a Banach algebra by the usual definition of the norm of a transformation $[7, \mathrm{p} .32]$ and with identity $l$. Let $\Omega(X)$ be the subset of $\mathscr{E}(X)$ consisting of the completely continuous transformations in $\mathscr{E}(\mathcal{X})$. It is well

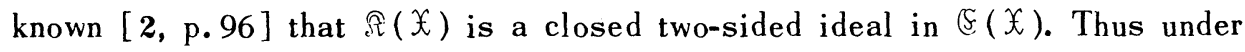
the usual definitions $[7$, p.472] the difference algebra $\mathscr{E}(\mathfrak{X})-\mathscr{X}(\mathfrak{X})$ is a 
Banach algebra. Let $\pi$ be the canonical homomorphism of $\mathscr{F}(\mathcal{X})$ into $\mathscr{E}(\mathfrak{X})$ -

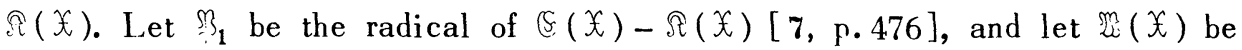
any closed two-sided ideal of $\mathscr{E}(X)$ contained in $\pi^{-1}\left(\Re_{1}\right)$ and containing $\mathscr{I}(X)$. Let $\tau$ be the canonical homomorphism of $\mathbb{E}(\mathfrak{X})$ onto $\mathscr{E}(\mathfrak{X})-\mathfrak{B}(\mathfrak{X})$.

2.1. LEMmA. $T \in \mathbb{E}(\mathfrak{X})$ has a left (right) inverse modulo $\mathfrak{X}(\mathfrak{X})$ if and only if $T$ has a left (right) inverse modulo $\Re(X)$.

Proof. Suppose that $T$ has a left inverse modulo $\mathfrak{N}(\mathfrak{X})$. Thus there exists $U \in \mathbb{E}(\mathfrak{X}), V \in \mathbb{W}(X)$ such that $U T=I+V$. Now $V \in \pi^{-1}\left(\mathfrak{P}_{1}\right)$ so that $I+V$ has a two-sided inverse $W$ modulo $\mathscr{\imath}(\mathfrak{X})$. Hence $W U$ is the desired left inverse of $T$ modulo $\AA(X)$.

It may be noted that since $\Re_{1}$ is closed in $\mathscr{E}(X)-\mathscr{X}(X)$ then $\pi^{-1}\left(\Re_{1}\right)$ is a closed two-sided ideal in $E(X)$.

2.2. Lemma. $T \in \mathfrak{E}(\mathfrak{X})$ has the properties that $T(X)$ is closed and its null-space is finite-dimensional if and only if $T$ takes each bounded set which is not conditionally compact onto a set which is not conditionally compact.

Lemma 2.2 is a rewording of $[15$, Lemma 3.1].

If the null-space of $T$ is finite-dimensional, its dimension is designated by nul $T$. A transformation with the properties of Lemma 2.2 is said in [15] to have property $A$.

2.3. LEMmA. $T \in \mathbb{E}(\mathfrak{X})$ has a two-sided inverse modulo $\mathbb{B}(\mathfrak{X})$ if and only if both $T$ and $T^{*}$ have property $A$.

Proof. By Lemma 2.1 we may take $\Re(X)$ for $\mathscr{X}(X)$. The result then follows immediately from the results of $[15, \S 5]$ ( see also $[1$, Theorem 1] and $[6]$ ).

If both $T$ and $T^{*}$ have property $A$ we define

$$
f(T)=\operatorname{nul} T^{*}-\operatorname{nul} T .
$$

Here $T^{*}$ is the adjoint of $T$ Let $\sqrt{2}$ be the set of all such transformations. By Lemma $2.3, \mathscr{S}_{2}$ is a semi-group.

2.4. Lemma. The function $f(T)$ is a continuous function on Fr. If $T$ and $U$ lie in the same component of $\tilde{S}$, then $f(T)=f(U)$. 
Proof. The continuity of $f$ follows from the work of Dieudonne' [4, proposition 4]; see also [15, Theorem 3.8] and [1, Theorem 4]. Since $f$ is integervalued, the second statement follows.

\subsection{Lemma. If $T \in \mathscr{S}$ and if $U-T \in \mathbb{W}(\mathfrak{X})$ then $U \in \mathfrak{S}$ and $f(T)=f(U)$.}

Proof. It is clear that $U$ has a two-sided inverse modulo $\mathscr{I}(X)$ if $T$ does, by Lemma 2.1. That $f(T)=f(U)$ follows from Lemma 2.4 since the set $T+\mathscr{W}(\mathfrak{X})$ is a connected subset of $\mathscr{T}_{2}$.

We adopt the following notation used by Rickart [12] for a Banach algebra. An element is left (right) regular provided that it possesses a left (right) inverse in the algebra. If the element is both left and right regular then it possesses a unique two-sided inverse and is said to be regular. For $(\mathfrak{F}(\mathfrak{X})$ we designate the sets of left regular, right regular, and regular elements by $\&$,

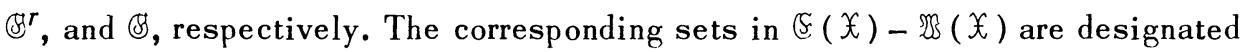
by $\&_{1} l$, \& ${ }_{1}^{r}$, and $\&_{1}$, respectively. In the foregoing notation, $\mathscr{L}_{2}=\tau^{-1}\left(\&_{1}\right)$.

Thus, by Lemmas 2.3 and 2.4, $f$ defines a mapping of $\&_{1}$ into the set of integers. This mapping will also be designated by $f$.

2.6. Lemma. Let $T \in \mathscr{F}_{2}, f(T)=0$. Then $T$ can be expressed as the sum $U+V$ where $U \in \mathbb{\&}, V \in \Omega(X)$.

Proof. This is given in [15, Corollary 3.11].

3. On the radical of $(\mathscr{E}(\mathfrak{X})-\mathfrak{W}(X)$. In view of Lemma 2.1 and the definition of the radical of $\mathbb{E}(X)-\mathscr{W}(X)$, the inverse image under $\tau$ of the radical of $\mathscr{E}(X)-W_{(X)}$ is the same set as $\pi^{-1}\left(\oiint_{1}\right)$, where $\Re_{1}$ is the radical of $\mathscr{E}(X)$ $\hat{\imath}(\mathfrak{X})$. In this section we determine the nature of $\pi^{-1}\left(\Re_{1}\right)$.

3.1. Lemma. Let $T \in \mathcal{E}(\mathfrak{X})$ be an isomorphism between $\mathfrak{X}$ and a proper closed linear manifold of $\mathfrak{X}$. Then there exists a sphere in $(\mathfrak{X}(\mathfrak{X})$ with center $T$ each of whose elements have this property.

Proof. By [4, proposition 1] there is a sphere $\subseteq$ about $T$ such that for all $U$ in $\widetilde{S}, U$ is bi-continuous. But $T$ is in the interior of the set of elements of $\mathscr{E}(X)$ which are not regular [14, Corollary 2.2]. Hence for each $U \in \widetilde{S}$ there is a proper closed linear manifold $\Re$ of $X$ such that $U$ is an isomorphism of $X$ onto $\Re$ if the radius of $\subseteq$ is sufficiently small.

3.2. Lemma. Let $T \in(\mathfrak{X})$ have range $\mathfrak{X}$ where $T$ is not one-to-one. Then 
there is a sphere in $\mathfrak{S}(\mathfrak{X})$ with center $T$ each of whose elements has these properties.

Proof. This is shown in the same way by use of [4, Theorem 1] and [14, Corollary 3.12].

3.3. Lemma. Let $T \in \mathbb{E}(\mathfrak{X})$. Suppose that $T\left(T^{*}\right)$ has property $A$ while $T^{*}(T)$ does not. Then $T$ can be expressed in the form $T_{1}+V$ where $V \in \Re(X)$ and $T_{1}$ is bi-continuous $\left(T_{1}(\mathfrak{X})=\mathfrak{X}\right)$.

Proof. This is contained in [15, Theorem 3.13].

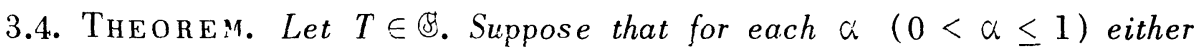
$T+\alpha U$ or $T^{*}+\alpha U^{*}$ has property $A$. Then $T+\alpha U \in \mathcal{F}_{2}(0 \leq \alpha \leq 1)$ and $f(T+U)=0$.

Proof. Note that $f(T)=0$. The set $\mathscr{S}_{2}=\pi^{-1}\left(\mathbb{B}_{1}\right)$ is open in $\mathscr{E}(X)$. Thus either all the $T+\alpha U(0 \leq \alpha \leq 1)$ are in $\mathcal{F}$ or there is a smallest number $\beta$ $(0<\beta \leq 1)$ such that $T+\beta U \notin \delta$. In the latter case one of $T+\beta U, T^{*}+\beta U^{*}$ has property $A$ but not the other. Suppose that $T+\beta U$ has property $A$. Then, by Lemma 3.3, $T+\beta U$ can be written in the form $T_{1}+V$, where $T_{1} \in \mathscr{F}(X)$ is bi-continuous and $V \in \mathcal{I}(\mathfrak{X})$. If $T_{1}(\mathfrak{X})=\mathfrak{X}$ then $T_{1} \in \mathbb{B}$ and thus $T+\beta U \in \mathscr{W}$, contrary to the above. Thus $T_{1}=T+\beta U-V$ an isomorphism between $\chi$ and a proper closed linear manifold of $\mathfrak{X}$. Consequently, by Lemma 3.1, if $0<\alpha<\beta$, and $\beta-\alpha$ is sufficiently small, then $T+\alpha U-V$ has this property. But for such

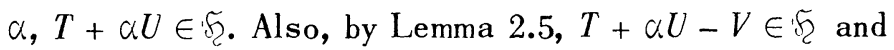

$$
f(T+\alpha U)=f(T+\alpha U-V) \text {. }
$$

Since

$$
\operatorname{nul}(T+\alpha U-V)=0 \text {, nul }\left(T^{*}+\alpha U^{*}-V^{*}\right)>0
$$

then

$$
f(T+\alpha U)>0
$$

However, since $f(T)=0$, by Lemma 2.4 we have

$$
f(T+\alpha U)=0
$$

This contradiction establishes the result if $T+\beta U$ has property $A$. If $T^{*}+\beta U^{*}$ has property $A$ then we proceed in a same way using dual results (Lemmas 3.2 
and 3.3) to see that for $\alpha<\beta$ and close to $\beta$,

$$
f(T+\alpha U)=0, \quad f(T+\alpha U)<0 .
$$

Thus we conclude that $T+\alpha U \in \mathbb{S}(0 \leq \alpha \leq 1)$. That

$$
f(T+U)=f(T)=0
$$

follows from Lemma 2.4.

3.5. THEOREM. The following formulas for $\pi^{-1}\left(\frac{m_{1}}{1}\right)$ hold:

(a) $\pi^{-1}\left(\dddot{\Re}_{1}\right)=\left\{U \in \mathbb{E}(\mathfrak{X}) \mid\right.$ for each $T \in \mathbb{B}$ either $T+U$ or $T^{*}+U^{*}$ has property $A\}$;

(b) $\pi^{-1}\left(\dddot{1}_{1}\right)=\{U \in \mathbb{E}(\mathfrak{X}) \mid T+U$ has property $A$ for each $T \in \mathbb{B}\}$;

(c) $\pi^{-1}\left(\dddot{\vartheta}_{1}\right)=\left\{U \in \mathbb{E}(\mathfrak{X}) \mid T^{*}+U^{*}\right.$ has property A for each $\left.T \in \mathbb{B}\right\}$.

Proof. If $T \in \mathbb{B}$ and $U \in \pi^{-1}\left(\dddot{丶}_{1}\right)$ then $\pi(T) \in \mathbb{B}_{1}$ and

$$
\pi(T+U)=\pi(T)+\pi(U) \in \mathbb{B}_{1},
$$

by the definition of $\Re_{1}$. Then $T+U \in \mathcal{F}_{2}$ and it follows that $\pi^{-1}\left(\Re_{1}\right)$ is contained in each of the sets on the right.

Let the set on the right side of (a) be denoted by $\widetilde{G}$. Then if $T \in \mathbb{B}, U \in \mathbb{G}$, $\alpha \neq 0$ a scalar, then $\alpha T+U$ or $\alpha T^{*}+U^{*}$ has property $A$. Hence, for each scalar $\alpha, T+\alpha U$ or $T^{*}+\alpha U^{*}$ has property $A$. Theorem 3.4 shows that $T+\alpha U \in f_{2}$ for all scalars $\alpha$. Next we show that if $W \in \mathbb{B}, U \in \mathcal{S}$ then $U W \in \Xi$. Both $W$ and $W^{*}$ have property $A$. Hence, by the nature of $\subseteq$ and $[15$, Theorem 3.4], for each $T \in \mathbb{B}$ either

$$
\left(T W^{-1}+U\right) W=T+U W
$$

has property $A$ or

$$
\mathbb{W} *\left[\left(T W^{\prime-1}\right) *+U^{*}\right]=T^{*}+(U W) *
$$

has property $A$. Hence $U W \in \Xi$.

Next let $U_{i} \in \mathcal{S}, i=1,2$. For each $T \in \mathbb{B}$, by the above $T+\alpha U_{1} \in \mathcal{F}_{\text {f }}$ for $0 \leq \alpha \leq 1$ and, by Theorem 3.4, $f\left(T+U_{1}\right)=0$. By Lemma 2.6, $T+U_{1}$ can be expressed in the form $T_{1}+V$, where $T_{1} \in \mathbb{B}$ and $V \in \mathscr{N}(\mathfrak{X})$. Likewise $T_{1}+U_{2} \in \mathscr{S}$ and so, by Lemma 2.5, 


$$
T_{1}+U_{2}+V=T+\left(U_{1}+U_{2}\right)
$$

is in $\mathscr{S}_{\text {. This shows that }} \subseteq$ is a linear manifold in $\mathscr{E}(\mathcal{X})$ with the further property that if $T_{1}, T_{2} \in \&$ and $U \in \widetilde{S}$ then $U\left(T_{1}-T_{2}\right) \in \Xi$. However, since $\mathscr{E}(\mathcal{X})$ is a Banach algebra, an arbitrary element $W \in \mathscr{E}(X)$ can be expressed as the difference of two regular elements. Thus $\widetilde{S}$ is a right ideal in $\mathscr{S}(\mathcal{X})$. Consequently $\pi(\Im)$ has the property that, for each $\pi(U) \in \pi(\Xi)$ and each $V$ in $\mathscr{S}(\mathfrak{X})-\mathscr{I}(\mathfrak{X})$, $\pi(I)+\pi(T) V \in \mathbb{B}_{1}$. Thus $\pi(\Im) \subset \Re_{1}$. This completes the proof for formula (a).

The same argument shows that the right sides of (b) and (c) are contained in $\pi^{-1}\left(\Re_{1}\right)$.

3.6. Corollary. Let $\Omega$ be a (left or right) ideal in $\mathbb{E}(X)$. Suppose that for each $T \in \Omega$, either $I+T$ or $I^{*}+T^{*}$ has property $A$. Then for each $T \in \Omega$, nul $(I+T)$ and $\mathrm{nul}\left(I^{*}+T^{*}\right)$ are finite and equal.

Proof. By Theorem 3.5, $\Omega \subset \pi^{-1}\left(\Re_{1}\right)$. Thus $I+T \in \mathscr{S}_{2}$ for each $T \in \Omega$. Since $\Omega$ is a linear manifold,

$$
f(I+T)=f(I)=0
$$

by Lemma 2.4 .

This is a direct generalization of Schauder's well-known result [13, p. 189] that if $U$ is completely continuous then

$$
\mathrm{nul}(I+U)=\operatorname{nul}\left(I^{*}+U^{*}\right)
$$

since the two-sided ideal $\Re(\mathfrak{X})$ fulfills the conditions of Corollary 3.6.

3.7. COROLLARY. The following statements are equivalent:

(1) $(\mathfrak{X})-\mathscr{X}(\mathfrak{X})$ is semi-simple;

(2) for $U \in \mathfrak{E}(\mathfrak{X}), U \in \Omega(X)$ if and only if $(T+U)(\mathfrak{X})$ is closed in $\mathfrak{X}$ and either nul $(T+U)$ or nul $\left(T^{*}+U^{*}\right)$ is finite for each $T$ regular in $(\mathcal{E}(\mathfrak{X})$.

Proof. Note that $\mathscr{E}(\mathfrak{X})-\Re(X)$ is semi-simple if and only if $\pi^{-1}\left(\Re_{1}\right)=\mathscr{I}(X)$. Also $(T+U)(X)$ is closed if and only if $\left(T^{*}+U^{*}\right)\left(X^{*}\right)$ is closed in $\mathfrak{X}^{*}$ [2, Chapt. 10]. Then Corollary 3.7 follows from Theorem 3.5 and Lemma 2.3.

If $\mathcal{X}$ is a separable Hilbert space then since, as shown by Calkin [ 3 , Theorem 1.4 ], $\mathscr{\complement}(\mathfrak{X})$ is a maximal, two-sided ideal in $(\mathfrak{X}(\mathfrak{X}),(1)$ holds. For spaces satisfying (1), (2) gives a necessary and sufficient condition for complete 
continuity which seems to be new (for sufficiency) even in the Hilbert space case.

4. A generalized Schauder nullity theorem. We give here the result (Theorem 4.5) discussed in $\S 1$. The preliminary material, it is felt, is of independent interest and is presented in greater generality than is absolutely necessary for our purposes.

We adopt the following notation. $B$ is a ring with an identity element $e$. $G$ is the set of regular elements of $B$ (the elements with a two-sided inverse). For each subgroup $G_{0}$ of $G$ let $\Im\left(G_{0}\right)$ be the set of "invariant translations" of $G_{0}$, namely the set of $x \in B$ such that $G_{0}+x=G_{0}$. It is clear that

$$
\Im\left(G_{0}\right)=\left\{x \in B \mid y \pm x \in G_{0} \text { for every } y \in G_{0}\right\} .
$$

In the ring $B$ we consider along with the usual algebraic operations also the "circle operation"

$$
x \circ y=x+y-x y .
$$

For information on this operation see [7, Chapter 22]. It is evident that $G_{0}$ n $\Im\left(G_{0}\right)$ is empty.

4.1. THЕОоем. For any subgroup $G_{0}$ of $G, \Im\left(G_{0}\right)$ is a subring of $B$ which is a group under the circle operation. Conversely if $R$ is a subring of $B$ which is a group under the circle operation then there exists a subgroup $G_{0}$ of $G$ such that $R=\Im\left(G_{0}\right)$. If $B$ is a Banach algebra then $\Im(G)$ is the radical of $B$.

Proof. It is clear that if $x \in \mathfrak{\Im}\left(G_{0}\right)$ then so does $-x$. Thus if $x_{1}$ and $x_{2}$ lie in $\Im\left(G_{0}\right)$, and $y \in G_{0}$, then both

$$
\left(y+x_{1}\right)+x_{2} \text { and }\left(y-x_{1}\right)-x_{2}
$$

lie in $G_{0}$, so that $x_{1}+x_{2} \in \Im\left(G_{0}\right)$. Next we show if $x \in \Im\left(G_{0}\right), y \in G_{0}$, then $y x \in \Im\left(G_{0}\right)$. For let $z \in G_{0}$. Then

$$
z \pm y z=y\left(y^{-1} z \pm x\right) \in G_{0}
$$

Similarly $x y \in \Im\left(G_{0}\right)$. Since

$$
y \pm x_{1} x_{2}=\left(y+x_{1}\right)\left(e \pm x_{2}\right) \bar{y} x_{2}-x_{1}
$$

it follows from the above that $x_{1} x_{2} \in \Im\left(G_{0}\right)$ if $x_{1}$ and $x_{2} \in \Im\left(G_{0}\right)$. Thus $\Im\left(G_{0}\right)$ is a subring of $B$. 
To see that $\Im\left(G_{0}\right)$ is a group under the circle operation note first that for $x_{1}, x_{2} \in \Im\left(G_{0}\right)$ we have

$$
x_{1} \circ x_{2}=x_{1}+x_{2}-x_{1} x_{2} \in \Im\left(G_{0}\right) .
$$

Now the set of all elements of $B$ with an inverse under the circle operation is a group with the zero element $\theta$ of $B$ as the identity element $[7, \mathrm{p} .456]$. Thus it is sufficient to show that $x_{1}$ has an inverse in $\Im\left(G_{0}\right)$ under this operation. Since $e-x_{1} \in G_{0} \subset G$ there exists an element $w \in B$ such that

$$
\left(e-x_{1}\right)(e-w)=(e-w)\left(e-x_{1}\right)=e .
$$

Then clearly $w$ is the inverse of $x_{1}$ under this operation. Let $y \in G_{0}$. Then, since

$$
x_{1} w=w x_{1}=x_{1}+w
$$

we have that

$$
(y \pm w)\left(e-x_{1}\right)=y \pm w-y x_{1} \mp w x_{1}=y\left(e-x_{1}\right) \mp x_{1}
$$

is an element of $G_{0}$. Since $\left(e-x_{1}\right) \in G_{0}$ it follows that $w \in \Im\left(G_{0}\right)$.

Next consider a subring $R$ which is a group under the circle operation. Let $G_{0}$ be the set of all elements of the form $e-x, x \in R$. If $x_{1}, x_{2} \in R$ then

$$
\left(e-x_{1}\right)\left(e-x_{2}\right)=e-x_{1} \circ x_{2} \in G_{0} \text {. }
$$

There exists $z \in R$ such that

$$
x_{1} \circ z=z \circ x_{1}=\theta \text {. }
$$

Then

$$
\left(e-x_{1}\right)(e-z)=(e-z)\left(e-x_{1}\right)=e
$$

so that $G_{0}$ is a group. We show that $\Im\left(G_{0}\right)=R$. Take $x \in \Im\left(G_{0}\right)$. Then $e-x \in G_{0}$, and, by the definition of $G_{0}, x \in R$. On the other hand if $x \in R, y \in G_{0}$ then we may write $y=e-x_{1}$, where

$$
x_{1} \in R \text { and } y \pm x=e-x_{1} \pm x \in G_{0}
$$

since $R$ is a ring. Thus $x \in \mathfrak{\Im}\left(G_{0}\right)$ and $\Im\left(G_{0}\right)=R$. 
Finally let $B$ be a Banach algebra. If $z$ is an arbitrary element of $B$ then since, for a sufficiently small scalar $\lambda$,

$$
e-\lambda z=w \in G
$$

we may write $z$ as the sum of two elements in $G$. By the above we see that for $x \in \Im(G)$, we have $z x \in \Im(G)$ and thus $e-z x \in G$. Hence $x$ lies in the (Jacobson) radical $Q$ of $B$. Conversely if $x \in Q$, then for each $w \in G$,

$$
w \pm x=w\left(e \pm w^{-1} x\right) \in G
$$

so that $x \in \Im(G)$. This completes the proof.

4.2. COROLLARY. In the notation of Theorem 4.1, $\Im\left(G_{0}\right)$ is a two-sided ideal in the subring $R\left(G_{0}\right)$ of $B$ generated by $G_{0}$ and lies in the radical $Q$ of $R\left(G_{0}\right)$. Examples exist for which $\Im\left(G_{0}\right)=Q$ and also for which $\Im\left(G_{0}\right) \neq Q$.

Proof. By the arguments of Theorem 4.1, if $y \in R\left(G_{0}\right)$ then $y x, x y \in \Im\left(G_{0}\right)$ for each $x \in \Im\left(G_{0}\right)$ so that $\Im\left(G_{0}\right)$ is a two-sided ideal of $R\left(G_{0}\right)$. Since $e-y x \in G_{0}$ for every $y \in R\left(G_{0}\right)$, and $G_{0}$ is contained in the set of regular elements of $R\left(G_{0}\right)$, $\Im\left(G_{0}\right) \subset Q$. By Theorem 4.1, if $B$ is a Banach algebra then $\widetilde{S}(G)=Q$. Take next for $B$ the ring of integers modulo 9. For $G_{0}$ take the set consisting of 1 and 8 . Here $R\left(G_{0}\right)=B$ and the radical $Q$ of $B$ is the set $\{0,3,6\}$. On the other hand $\Im\left(G_{0}\right)$ consists of the zero element alone.

Following Kaplansky [8, p. 153] we call $B$ a metric ring if to each element $x$ there is associated a real number $|x|$ such that

$$
|\theta|=0,|x|>0 \text { if } x \neq \theta,|-x|=|x|,|x+y| \leq|x|+|y|,|x y| \leq|x||y| \text {. }
$$

Here $|x-y|$ is the metric of $B$. In this context the sets $\Im\left(G_{0}\right)$ possesses certain topological properties. (The metric ring to which the theory is applied is $(\mathcal{E}(\mathfrak{X})-\mathscr{S}(\mathfrak{X}))$.

4.3. LEMmA. If $G_{0}$ is open then $\widetilde{\Im}\left(G_{0}\right)$ is closed. The following statements are equivalent.

(1) $\widetilde{\Im}\left(G_{0}\right) \subset \bar{G}_{0}$.

(2) $0=\inf |y|, y \in G_{0}$.

(3) $\Im\left(G_{0}\right) \cap \overline{G_{0}}$ is nonempty.

Proof. Let $G_{0}$ be open. Suppose that $x_{n} \in \Im\left(G_{0}\right)(n=1,2,3, \ldots)$ and 
that $x_{n} \longrightarrow x$. Given any $y \in G_{0}$ there exists a sphere $S$ of radius, say, $r>0$ about $y$ such that $S \subset G_{0}$. Consequently $S \pm x_{n} \subset G_{0}$ for each $n$. Take $n$ so large that $\left|x-x_{n}\right|<r$. Then for such an integer $n, y \pm\left(x-x_{n}\right) \in S$ and thus

$$
y \pm x=y \pm\left(x-x_{n}\right) \mp x_{n} \in G_{0} .
$$

Hence $x \in \mathfrak{\Im}\left(G_{0}\right)$.

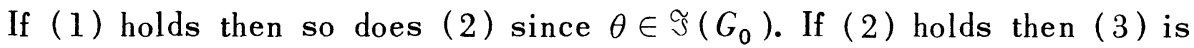
clear for the same reason. Suppose that (3) holds. Let

$$
w \in \Im\left(G_{0}\right) \cap \bar{G}_{0}, w=\lim y_{n}, y_{n} \in G_{0} \text {. }
$$

By Theorem 4.1, $w \circ x \in \mathfrak{S}\left(G_{0}\right)$ for each $x \in \mathfrak{\Im}\left(G_{0}\right)$. But

$$
w \circ x=\lim \left(x+y_{n}-y_{n} x\right)
$$

and by Theorem 4.1, $y_{n}+x-y_{n} x \in G_{0}$. Hence $w \circ x \in \overline{G_{0}}$. By Theorem 4.1 again there exists an element $z$ in $\Im\left(G_{0}\right)$ such that $w \circ z=\theta$. Inasmuch as $z \circ x \in \mathfrak{s}\left(G_{0}\right)$, by the above

$$
w \circ(z \circ x)=(w \circ z) \circ x=x
$$

lies in $\overline{G_{0}}$.

For the group $G_{0}$ in the metric ring $B$ let $G_{0 p}$ be the principal component, that is, that which contains $e$. Arguments of Hille $\left[7\right.$, p.93] show that $G_{0 p}$ is a subgroup of $G_{0}$.

4.4. LEммA. If $\Im\left(G_{0 p}\right) \subset \bar{G}_{0 p}$ then $\Im\left(G_{0}\right)$ is connected and $\Im\left(G_{0}\right) \subset \bar{G}_{0 p}$. If $\widetilde{\Im}\left(G_{0}\right)$ is connected, then $\Im\left(G_{0}\right) \subset \Im\left(G_{0 p}\right)$.

Proof. Suppose that $\Im\left(G_{0 p}\right) \subset \bar{G}_{0 p}$. Then by Lemma 4.3, $\theta \in \bar{G}_{0 p}$. Take $x \in \mathfrak{S}\left(G_{0}\right)$. The set $x G_{0 p}$, being a continuous image of a connected set, is connected; moreover, $x G_{0 p}$ lies in $\widetilde{\Im}\left(G_{0}\right)$ by Corollary 4.2. Since $\theta$ lies in the closure of $x G_{0 p}$, the set

$$
F=x G_{0 p} \cup\{\theta\}
$$

is a connected subset of $\widetilde{\Im}\left(G_{0}\right)$ which contains $x$ and $\theta$. Hence each element of $\widetilde{\Im}\left(G_{0}\right)$ lies in a connected subset containing $\theta$. Thus $\Im\left(G_{0}\right)$ is connected.

Suppose that $\Im\left(G_{0}\right)$ is connected. Then for each $z \in G_{0 p}, z+\Im\left(G_{0}\right)$ is a connected subset of $G_{0}$ containing $z$. Hence 


$$
z+\Im\left(G_{0}\right) \subset G_{0 p} \text { and } \Im\left(G_{0}\right) \subset \Im\left(G_{0 p}\right)
$$

In the statement of the following theorem, the group to which the symbol $\mathfrak{\Im}$ is applied lies in the Banach algebra $\&(X)-\pi^{-1}\left(\Re_{1}\right)$.

4.5. THEOREM. Let $\subseteq$ be any set in $\mathscr{E}(X)$ containing the identity 1 . Let $\pi$ and $\tau$ be the canonical homomorphisms of $\mathscr{E}(\mathfrak{X})$ onto $\mathscr{E}(X)-\Re(X)$ and $\mathscr{E}(X)-\pi^{-1}\left(\Re_{1}\right)$, respectively. Suppose that $\pi(\mathcal{S})$ is a multiplicative group in $\mathscr{E}(\mathfrak{X})-\Omega(X)$ and that the closure of the component of $\subseteq$ containing I contains

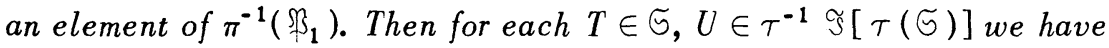

$$
f(T)=f(T+U) .
$$

Furthermore, $\tau^{-1} \Im[\tau(\widetilde{S})] \supset \pi^{-1}\left(\Re_{1}\right)$, and is the inverse image under $\pi$ of a subring of $(\mathfrak{X})-\mathfrak{X}(\mathfrak{X})$ which is a group under the circle operation.

Proof. Consider $\tau(\mathbb{S})$. By Lemma 2.1 it is a subgroup of the set of regular elements $\mathscr{B}_{1}$ of $\mathscr{E}(\mathfrak{X})-\pi^{-1}\left(\Re_{1}\right)$. Since $\tau$ is continuous, by our hypothesis the principal component of $\tau(\subseteq)$ contains the zero element of $\mathscr{E}(X)-\pi^{-1}\left(\Re_{1}\right)$ in its closure. Hence in this algebra, by Lemmas 4.3 and 4.4, $\Im[\tau(\subseteq)]$ is con-

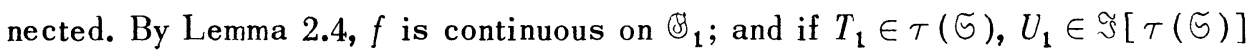
then since $T_{1}$ and $T_{1}+U_{1}$ lie in the same component of $\mathscr{B}_{1}$, we have

$$
f\left(T_{1}+U_{1}\right)=f\left(T_{1}\right) \text {. }
$$

Thus $f(T+U)=f(T)$ if $T \in \widetilde{S}$ and $U \in \tau^{-1}[\Im(\tau(\widetilde{S})]$.

Let

$$
\tau^{-1} \Im[\tau(\widetilde{S})]=\widetilde{S}_{1} \text { and } \pi\left(\widetilde{\Xi}_{1}\right)=\widetilde{S}_{2}
$$

Clearly $\pi^{-1}\left(\widetilde{S}_{2}\right)=\widetilde{S}_{1}$ since $\widetilde{S}_{1} \supset \Re(X)$ which is the kernel of $\pi$. By Theorem 4.1, $\mathfrak{\Im}[\mathcal{T}(\mathfrak{S})]$ is a subring of $\mathscr{S}(\mathfrak{X})-\pi^{-1}\left(\Re_{1}\right)$ which is a group under the circle Operation. Then $\widetilde{S}_{1}$ is a subring of $\mathscr{E}(\mathfrak{X})$, and $\widetilde{S}_{2}$ a subring of $\mathscr{E}(\mathfrak{X})-\mathscr{\mathcal { X }}(\mathfrak{X})$. We next show that $\widetilde{S}_{2}$ is a group under the circle operation. $A s \widetilde{S}_{2}$ is a subring, it is closed under that operation. Let $T_{1} \in \widetilde{S}_{2}, T_{1}=\pi(T), T \in \overleftarrow{S}_{1}$. Then there exists $V \in \widetilde{S}_{1}$ such that

$$
[\tau(I)-\tau(V)][\tau(I)-\tau(T)]=[\tau(I)-\tau(T)][\tau(I)-\tau(V)]=\tau(I) .
$$

Then by Lemma $2.1, I-T$ has a two-sided inverse $I-W$ modulo $\Omega(X)$. Since

$$
T_{1} \circ \pi(W)=\pi(\mathbb{W}) \circ T_{1}=0
$$


it suffices to show that $\pi(\mathbb{W}) \in \widetilde{S}_{2}$. Now $\tau(\mathbb{W})=\tau(V)$ since the two-sided inverse of $\tau(I-T)$ in $\mathscr{E}(X)-\pi^{-1}\left(\S_{1}\right)$ is unique. Therefore $W \in \widetilde{S}_{1}$ and thus $\pi(W) \in \widetilde{G}_{2}$.

5. Functionals on semi-groups. Atkinson [1] has shown that on $\sqrt{2}$ the equation

$$
f(T U)=f(T)+f(U)
$$

is valid. By an entirely different analysis we show how such functionals can be obtained in a semi-group and then apply the results to 5 .

5.1. Notation. Let $S$ be any semi-group, the product of two elements $x, y$ in $S$ being denoted by $x y$. Let $g$ and $g *$ be real-valued functions defined on $S$, where

$$
\begin{aligned}
& g\left(x_{2}\right) \leq g\left(x_{1} x_{2}\right) \leq g\left(x_{1}\right)+g\left(x_{2}\right) \\
& g *\left(x_{1}\right) \leq g^{*}\left(x_{1} x_{2}\right) \leq g^{*}\left(x_{1}\right)+g^{*}\left(x_{2}\right)
\end{aligned}
$$

for all $x_{1}, x_{2}$ in $S$. Let

$$
h(x)=g^{*}(x)-g(x),
$$

and let $S_{+}\left(S_{-}\right)$be the subset of $S$ for which $h(x) \geq 0(h(x) \leq 0)$. Suppose that there is a reflexive and symmetric relation $\sim$ on $S$ defined for certain pairs of elements of $S$ such that $x \sim y$ implies $h(x)=h(y)$, and where for each $x \in S$ there exists $y \in S, x \sim y$ with either $g(y)=0$ or $g^{*}(y)=0$, The relation $\sim$ need not be transitive. Since $g$ and $g^{*}$ are nonnegative on $S$ it follows that the existence of $y, x \sim y$, where $g(y)=0\left(g^{*}(y)=0\right)$, is equivalent to $x \in S_{+}\left(x \in S_{-}\right)$.

5.2. The OREм. Suppose that, in the notation of 5.1,

(a) $x_{i} \sim z_{i}(i=1,2)$ implies that $h\left(x_{1} x_{2}\right)=h\left(z_{1} z_{2}\right)$ holds. Then the formula

$$
h\left(x_{1} x_{2}\right)=h\left(x_{1}\right)+h\left(x_{2}\right)
$$

is valid either for all $x_{1} \in S_{+}$or for all $x_{2} \in S_{-}$. If also

(b) there exist $y_{1}, y_{2}$ in $S$, where $h\left(y_{1}\right)>0$ and $h\left(y_{2}\right)<0$, then formula (2) is valid on $S$.

Formula (2) is valid on $S$ if (a) holds and 
$\left(c_{1}\right)$ for each $x \in S_{+}$there exists $y \in S$ such that $x y \in S_{-}$,

$\left(c_{2}\right)$ for each $x \in S_{-}$there exists $y \in S$ such that $y x \in S_{+}$.

Proof. We remark that (a) is a necessary condition for (2) since, from (2),

$$
h\left(x_{1} x_{2}\right)=h\left(x_{1}\right)+h\left(x_{2}\right)=h\left(z_{1}\right)+h\left(z_{2}\right)=h\left(z_{1} z_{2}\right) .
$$

From ( 1 ) we obtain

$$
g^{*}\left(x_{1}\right)-g\left(x_{1}\right)-g\left(x_{2}\right) \leq g^{*}\left(x_{1} x_{2}\right)-g\left(x_{1} x_{2}\right) \leq g^{*}\left(x_{1}\right)+g^{*}\left(x_{2}\right)-g\left(x_{2}\right)
$$

or

$$
h\left(x_{1}\right)-g\left(x_{2}\right) \leq h\left(x_{1} x_{2}\right) \leq h\left(x_{2}\right)+g^{*}\left(x_{1}\right)
$$

Now suppose that (a) holds. Then

$$
\begin{array}{ll}
h\left(x_{1}\right) \leq h\left(x_{1} x_{2}\right) \leq h\left(x_{1}\right)+h\left(x_{2}\right) & x_{1}, x_{2} \in S_{+}, \\
h\left(x_{1}\right)+h\left(x_{2}\right) \leq h\left(x_{1} x_{2}\right) \leq h\left(x_{2}\right) & x_{1}, x_{2} \in S_{-}, \\
h\left(x_{1} x_{2}\right)=h\left(x_{1}\right)+h\left(x_{2}\right) & x_{1} \in S_{+}, x_{2} \in S_{-} .
\end{array}
$$

To show (4) we may assume that

$$
g\left(x_{i}\right)=0, g^{*}\left(x_{i}\right)=h\left(x_{i}\right) \quad(i=1,2) .
$$

Then (4) follows from (3). For (5) we may assume that

$$
-g\left(x_{i}\right)=h\left(x_{i}\right), g^{*}\left(x_{i}\right)=0
$$

and again use (3). In the last situation, ( 3 ) yields

$$
h\left(x_{1}\right)+h\left(x_{2}\right) \leq h\left(x_{1} x_{2}\right) \leq h\left(x_{1}\right)+h\left(x_{2}\right) .
$$

Next we observe that $\left(c_{1}\right)$ and $\left(c_{2}\right)$ cannot both be false. If, for example, $\left(c_{1}\right)$ is false then for some $x_{1} \in S_{+}$we have $x_{1} y \in S_{+}$for all $y \in S$, which yields $\left(c_{2}\right)$.

Suppose now that (a) and $\left(c_{2}\right)$ hold. We show that (2) holds for all $x_{1}, x_{2}$ where $x_{2} \in S_{\text {. }}$ By (6) we may suppose that $x_{1} \in S_{\text {-. }}$ There exists $w \in S$ such

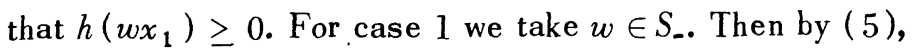

$$
h(w)+h\left(x_{1}\right) \leq h\left(w x_{1}\right) \leq h\left(x_{1}\right) \leq 0 .
$$


This implies that $h\left(x_{1}\right)=0$. Then (2) follows from (6). For case 2 we take $w \in S_{+}$. This gives, by (6),

$$
\begin{gathered}
h\left(w x_{1}\right)=h(w)+h\left(x_{1}\right), \\
h\left(w x_{1} x_{2}\right)=h\left(w x_{1}\right)+h\left(x_{2}\right) .
\end{gathered}
$$

Now ( 5 ) shows that $x_{1} x_{2} \in S_{-}$. Then, by (6),

$$
h\left(w x_{1} x_{2}\right)=h(w)+h\left(x_{1} x_{2}\right) .
$$

A combination of ( 7 ), ( 8 ), and (9) yields (2).

Suppose next that $(a)$ and $\left(c_{1}\right)$ hold. Entirely analogous arguments using (4) in place of (5) show that (2) holds for all $x_{1}, x_{2}$ where $x_{1} \in S_{+}$.

Now assume $(a)$ and $(b)$. We show that $\left(c_{1}\right)$ and $\left(c_{2}\right)$ hold. If $\left(c_{1}\right)$ does not hold then $\left(c_{2}\right)$ must hold and there exists $x \in S_{+}$such that $x y \in S_{+}$for all $y \in S$. Select $y$ such that $h(y)<0$. By $(\mathrm{a})$ and $\left(\mathrm{c}_{2}\right)$ and the above, $h\left(y^{n}\right)=$ $n h(y)$ for any positive integer $n$ and thus $y^{n} \in S_{\text {. }}$. Also

$$
0 \leq h\left(x y^{n}\right)=h(x)+n h(y) \text {. }
$$

This is impossible if $n$ is chosen sufficiently large. Thus $\left(c_{1}\right)$ holds. Similarly $\left(c_{2}\right)$ holds.

To conclude the proof we show that $(a),\left(c_{1}\right)$, and $\left(c_{2}\right)$ imply (2). By the above our assumptions give the validity of (2) for any pair $x_{1}, x_{2}$ where either $x_{1} \in S_{+}$or $x_{2} \in S_{-}$. The remaining case involves $x_{1} \in S_{-}$and $x_{2} \in S_{+}$. We may select, by $\left(\mathrm{c}_{2}\right), w \in S$ such that $w x_{1} \in S_{+}$. If $w \in S_{-}$then, as shown above, $h\left(x_{1}\right)=0$ so that $(2)$ is valid for $x_{1}, x_{2}$. Supposing that $w \in S_{+}$, we obtain (7), (8), and (9), which again yield (2) for $x_{1}, x_{2}$.

We return to $\mathscr{E}(X)$ and start with the following simple result:

5.3. Lемma. Let $T_{i} \in \mathfrak{S}(X)(i=1,2)$ have finite nullity. Then

$$
\operatorname{nul}\left(T_{2}\right) \leq \operatorname{nul}\left(T_{1} T_{2}\right) \leq \operatorname{nul}\left(T_{1}\right)+\operatorname{nul}\left(T_{2}\right)
$$

This follows from the fact, readily established, that

$$
\operatorname{nul}\left(T_{1} T_{2}\right)=\operatorname{nul}\left(T_{2}\right)+\operatorname{dim}\left[T_{2}(\nVdash) \cap T_{1}^{-1}(0)\right]
$$

5.4. Lemma. Suppose that $T \in \mathcal{F}_{2}$ and $f(T) \geq 0(\leq 0)$. Then there exists $V \in \mathscr{S}$ such that $V-T \in \mathcal{\Omega}(\mathfrak{X}), f(T)=f(V)$, and nul $(V)=0\left(\operatorname{nul}\left(V^{*}\right)=0\right)$. 
The existence of the transformation $V$ with the indicated property of the nullity follows from [15, Theorem 3.13]. That $f(T)=f(V)$ follows from Lemma 2.5 .

5.5. Corollary. Let $T_{i} \in \mathscr{S}_{2}(i=1,2)$. Then $f\left(T_{1} T_{2}\right)=f\left(T_{1}\right)+f\left(T_{2}\right)$, and $f$ defines a homomorphis $m$ of the group of regular elements of $(\mathfrak{E}(X)-\Re(X)$ into the additive group of integers.

We show that this result of Atkinson follows from the above. In the notation of 5.1 , set

$$
S=\sqrt[5]{2}, g^{*}(T)=\operatorname{nul}\left(T^{*}\right), g(T)=\operatorname{nul}(T)
$$

Since

$$
\left(T_{1} T_{2}\right)^{*}=T_{2}^{*} T_{1}^{*}
$$

Lemma 5.3 shows that formula ( 1 ) is valid. For the relation $T_{1} \sim T_{2}$ we take $T_{1}-T_{2} \in \Omega(X)$. Lemmas $3.2,2.4$, and 5.4 and the relation

$$
f(T)=\operatorname{nul}\left(T^{*}\right)-\operatorname{nul}(T)
$$

show that Theorem 5.2 may be applied to give the first conclusion. The second conclusion is an immediate consequence.

Following ideas of Mackey [10, p. 171] we shall say that the Banach space $X$ is stable if there exists a continuous isomorphism of $\mathfrak{X}$ onto a closed subspace $\mathfrak{X}_{1}$ of deficiency one. We say that $X$ is stable-like if there exists a continuous isomorphism of $\mathfrak{X}$ onto a closed subspace $X_{1}$ of finite deficiency.

5.6. THEOREM. The functional $f$ is non-trivial if and only if $\mathfrak{X}$ is stablelike.

Proof. If $\mathfrak{X}$ is stable-like, consider the isomorphism $T$ of $\nVdash$ onto $\mathfrak{X}_{1}$ of deficiency $n$. Then nul $\left(T^{*}\right)=n$ and nul $(T)=0$, so that $f(T)=n$.

Suppose that $f$ is non-trivial. Then there exists $T \in 1 \sqrt{2}$ such that $f(T) \neq 0$. Since $T$ has a two-sided inverse $V$ modulo $\Omega(X)$, and $f(V)=-f(T)$ by Corollary 5.5, we may assume $f(T)=n>0$. By Lemma 5.4, there exists a bicontinuous isomorphism $U$ where nul $\left(U^{*}\right)=n$. Then $U(X)$ is a closed subspace of deficiency $n$.

Whether or not every infinite-dimensional Banach space must be stable or even stable-like seems to be an open question (see [10, p. 205]). This subject 
is pursued a bit further in Theorem 6.7 and 6.9.

If $\chi$ is finite-dimensional then (10) can be replaced by the more specific rule, known as Sylvester's law of nullity $[9, \mathrm{p} .11]$ which states that

$$
\max \left[\operatorname{nul}\left(T_{1}\right), \operatorname{nul}\left(T_{2}\right)\right] \leq \operatorname{nul}\left(T_{1} T_{2}\right) \leq \operatorname{nul}\left(T_{1}\right)+\operatorname{nul}\left(T_{2}\right)
$$

We show that the validity of Sylvester's rule for all $T_{i} \in \sqrt{2}$ where $X$ is infinitedimensional implies that $\chi$ is not stable-like. For suppose otherwise. Consider

$$
T_{2} \in \mathscr{F}_{2}, f\left(T_{2}\right)=n>0, \operatorname{nul}\left(T_{2}\right)=0 \text {. }
$$

Then by $\left[14\right.$, Theorem 3.15] there exists $T_{1} \in \mathscr{E}(X)$ such that $T_{1} T_{2}=I$. Since $I$ and $T_{2} \in \mathcal{F}_{2}$, by [15, Theorem 5.4] we see that $T_{1} \in \mathcal{F}_{2}$. By Sylvester's rule, nul $\left(T_{1}\right)=0$, so that $T_{1}$ is regular in $\left(\mathscr{E}(\mathfrak{X})\right.$ and therefore so is $T_{2}$, which is a contradiction.

Another generalization of Schauder's theorem may be obtained as follows. Yosida and Kakutani [16] have considered the collection $\supseteqq(X)$ of all quasicompletely continuous transformations in $\mathscr{F}(X)$ i.e. the class of all $T \in \mathcal{E}(X)$ such that there exists $V \in \mathfrak{I}(\mathfrak{X})$ and an integer $n$ such that $\left\|T^{n}-V\right\|<1$.

5.7. ThE OREM. Let $T \in \mathcal{W}$, and let $V$ be a two-sided inverse of $T$ modulo $\Omega(X)$. Suppose that there exists $\mathbb{W} \in \pi^{-1}\left(\Re_{1}\right)$ and an integer $m$ such that $V^{m} U-\mathbb{W} \in \mathfrak{D}(X)$. Then $T^{m}+U \in \mathfrak{S}$, and

$$
f\left(T^{m}+U\right)=m f(T) \text {. }
$$

Proof. Let $V^{m} U=R_{1}$ and $R_{1}-\mathbb{W}=R_{2}$. By hypothesis there is an integer $n$ such that $I-R_{2}^{n}$ is of the form $S_{1}^{-}+S_{2}$, where $S_{1} \in \mathbb{B}$ and $S_{2} \in \mathfrak{A}(\mathfrak{X})$. Since $\pi^{-1}\left(\Re_{1}\right)$ is a two-sided ideal, there exists $S_{3} \in \pi^{-1}\left(\Re_{1}\right)$ such that

$$
I-R_{1}^{n}=S_{1}+S_{3}
$$

But, by Lemma 2.5, $S_{1}+S_{3} \in \sqrt[5]{ }$. Therefore $l-R_{1}^{n}$ has a two-sided inverse modulo $\Re(\chi)$. Since

$$
I-R_{1}^{n}=\left(I-R_{1}\right)\left(I+R_{1}+\cdots+R_{1}^{n-1}\right)=\left(I+R_{1}+\cdots+R_{1}^{n-1}\right)\left(I-R_{1}\right)
$$

then $I-R_{1} \in \mathscr{S}_{2}$. Since the hypothesis on $U$ is satisfied by all $\alpha U,|\alpha| \leq 1$, it follows from Theorem 3.4 that

$$
f\left(I-R_{1}\right)=f\left(I+R_{1}\right)=0 .
$$


Applying Corollary 5.5, we obtain

$$
f\left(T^{m}+U\right)=f\left[T^{m}\left(I+R_{1}\right)\right]=m f(T) .
$$

6. On the images of left and right regular elements. We make here a detailed study of the images of the sets \&, \&l, and \&r under $\pi$. In view of Lemma 2.1, the results also hold for the mapping $\tau$. In particular, we show the following:

6.1. ThEOREM. The canonical homomorphism $\pi$ has the following properties:

( 1 ) $\pi\left(B^{l} \cup B^{r}\right)=\pi\left(B^{l}\right) \cup \pi\left(B^{r}\right)=B_{1}^{l} \cup B_{1}^{r}$;

(2) $\pi(B)=\pi\left(B^{l}\right) \cap \pi\left(B^{r}\right)$;

(3) the sets $\pi$ (\&), $\pi\left(\mathbb{B}^{l}\right)$, and $\pi\left(\mathbb{B}^{r}\right)$ are open and closed in the sets $\mathbb{B}_{1}$, $\mathrm{BS}_{1}^{l}$, and $\mathrm{Br}_{1}^{r}$, respectively;

(4) $\pi\left(\right.$ (B) is a normal subgroup of $B_{1}$; either $\pi(B)=B_{1}$ or $B_{1} / \pi($ (B) is isomorphic, as a topological group, to the additive group of integers in the discrete topology.

The interest of $(1)$ lies in the fact that if $\chi$ is stable-like, then $\pi\left(B^{l}\right) \neq \&_{1}^{l}$ and $\pi\left(B^{r}\right) \neq \otimes_{1}^{r}$ (see Lemma 6.3). And for (2), even though $B=B^{l} \cap \& S^{r}$ this does not of itself imply that

$$
\pi\left(B^{l} \cap B^{r}\right)=\pi\left(B^{l}\right) \cap \pi\left(B^{r}\right) \text {. }
$$

In the course of the proof the following notation is used. $12_{0}$ is the subset of $\sqrt{2}$ consisting of those $T$ for which $f(T)=0$ and $\mathscr{L}_{+}\left(\mathscr{S}_{-}\right)$of those $T$ for which $f(T)>0(f(T)<0)$. The minus sign for sets in $(\mathscr{E}(\mathfrak{X})-\mathfrak{I}(\mathfrak{X})$ is used in the set-theoretic sense. From the definitions we have $\pi(52)=\mathscr{B}_{1}$.

The following lemmas are part of the proof of Theorem 6.1.

6.2. LEMmA. $\pi(\&)=\left\{T_{1} \in \mathbb{E}(X)-\Re(X) \mid \pi^{-1}\left(T_{1}\right) \subset \mathscr{S}_{0}\right\}$, and $\pi(\&)=\pi\left(\mathscr{S}_{0}\right)$.

Proof. The second statement follows immediately from the first. Suppose that

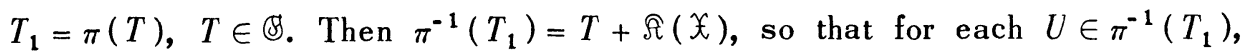
$f(U)=f(T)$ by Lemma 2.5. Since $f(T)=0$, we see that $\pi(B)$ is contained in the right-hand set. Next assume that $T_{1}$ is in the right-hand set. Let $\pi(T)=T_{1}$. Then $T \in \mathscr{F}_{0}$, and $f(T)=0$. By Lemma 2.6 there exists $V \in \mathfrak{I}(\mathfrak{X})$ such that $T+V \in$ \&. But $\pi(T+V)=T_{1}$. 


\subsection{LEMMA. $\pi\left(\mathbb{B}^{l}\right)=\mathbb{B}_{1}^{l}-\pi\left(\mathscr{S}_{-}\right)$.}

Proof. Clearly $\pi\left(\mathbb{B}^{l}\right) \subset \mathbb{B}_{1}^{l}$. We shall show that $\pi\left(\mathbb{B}^{l}\right) \cap \pi\left(\mathscr{\zeta}_{-}\right)$is empty. Suppose contrariwise that $T_{1} \in \pi(\mathbb{S} l) \cap \pi\left(\mathscr{F}_{-}\right)$. Then there exists $T \in \mathbb{B} l, U \in \mathscr{L}_{-}$such that $\pi(T)=\pi(U)=T_{1}$. Then there exists $\mathbb{W} \in \Re(X)$ such that $T=U+\mathbb{W}$. Hence, by Lemma 2.5, $f(T)=f(U)<0$. But from the definition of $f$, nul $(T)>0$. Therefore $T$ cannot be one-to-one and this contradicts $T \in \mathbb{B} l$. We conclude that $\pi\left(\otimes^{l}\right) \subset \otimes_{1}^{l}-\pi\left(\delta_{2}\right)$.

Suppose that $T_{1} \in \mathbb{S}_{1}^{l}-\pi\left(S_{-}\right)$and $\pi(T)=T_{1}$. By [15, Theorem 5.4], $T$ has property $A$. Since $T \notin \mathfrak{F}_{-}$, either nul $\left(T^{*}\right)$ is not finite or nul $\left(T^{*}\right)<\propto$ and $f(T) \geq 0$. Then by [15, Theorem 3.13] there exists $V \in \Omega(X)$ such that $T+V$ is a bi-continuous mapping of $\mathfrak{X}$ into $\mathfrak{X}$. Moreover, by [15, Theorems 5.3 and 5.4], there exists a projection of $\mathfrak{X}$ onto $(T+V)(\mathfrak{X})$. Therefore, by [14, Theorem 3.15], $T+V \in$ \&l. However, $\pi(T+V)=\pi(T)=T_{1}$. Thus \&l $-\pi\left(1 \xi_{-}\right) \subset$ $\pi\left(B^{l}\right)$.

6.4. LeMMA. $\pi(\otimes r)=\&{ }_{1}^{r}-\pi\left(\mathscr{S}_{+}\right)$.

In references cited in the proof of Lemma 6.3, dual results exist to those used in 6.3 which enable one to conduct the proof in the same way.

6.5. LEMMA. $\pi\left(\mathscr{S}_{-}\right) \subset \pi\left(\otimes^{r}\right)$ and $\pi\left(\mathscr{S}_{+}\right) \subset \pi\left(\otimes^{l}\right)$.

Proof. Suppose that $T \in \mathcal{S}_{2}$. By [15, Theorem 3.13] there exists $V \in \Re(\mathfrak{X})$ such that $(T+V)(\mathfrak{X})=\mathfrak{X}$. Also, by Lemma 2.4, nul $(T+V)<\infty$. Hence [14, Theorem 3.18] shows that $T+V \in \mathbb{B} r$. However, $\pi(T+V)=\pi(T)$. The other statement is proved using dual results.

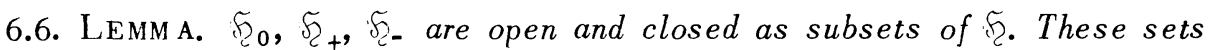
are disjoint.

Proof. Since $f(T)$, by Lemma 2.5, is a continuous integral-valued function on $5_{2}$, the sets are open and closed subsets of $5_{2}$.

We turn now to the statements of Theorem 6.1.

Consider (1). By Lemmas 6.3 and 6.4,

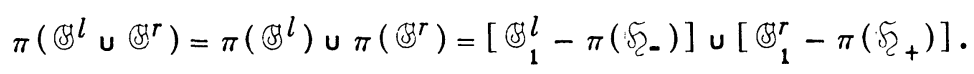

By Lemma 6.5,

$$
\pi\left(\mathscr{S}_{-}\right) \subset \pi\left(\mathbb{B}^{r}\right), \pi\left(\mathscr{S}_{+}\right) \subset \pi\left(\text { \& }^{l}\right),
$$


so that

$$
\pi\left(\mathbb{B}^{l}\right) \cup \pi\left(B^{r}\right)=\mathbb{B}_{1}^{l} \cup \otimes_{1}^{r} \text {. }
$$

As for (2), note first that $\pi(\mathbb{F})=\pi\left(\mathcal{S}_{0}\right)$ by Lemma 6.2. By Lemmas 6.3 and 6.4 ,

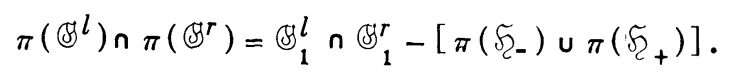

But $\mathbb{B}_{1} l \cap \mathbb{B} r=\mathbb{B}{ }_{1}=\pi\left(\mathscr{S}_{2}\right)$. Also the sets $\pi\left(\mathscr{S}_{+}\right), \pi\left(\mathscr{F}_{-}\right)$and $\pi\left(\mathscr{S}_{0}\right)$ are disjoint since if, for example, $T_{1} \in \pi\left(\mathcal{F}_{+}\right) \cap \pi\left(\mathcal{S}_{-}\right), T_{1}=\pi(T), T \in \mathcal{F}_{+}$and $T_{1}=\pi(V)$, $V \in \mathscr{S}_{-}$, then $\pi(T-V)=0$ so that $T-V \in \mathbb{R}(\mathfrak{X})$; whence, by Lemma 2.4, $f(T)=f(V)$ which is impossible. Hence

$$
\pi\left(B^{l}\right) \cap \pi\left(\otimes^{r}\right)=\pi\left(\mathscr{S}_{2}\right)-\left[\pi\left(\mathscr{S}_{-}\right) \cup \pi\left(\mathscr{S}_{+}\right)\right]=\pi\left(\mathscr{S}_{2}\right)=\pi(\&) .
$$

The mapping $\pi$ is a continuous linear mapping of the Banach algebra $\mathscr{E}(\mathfrak{X})$ onto the Banach algebra $\mathscr{E}(\mathfrak{X})-\Omega(X)$. Consequently it takes open sets into

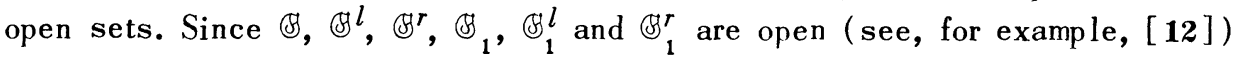
the statement of $(3)$ on openness follows. Likewise, from Lemma 6.6, $\pi\left(r_{2}\right)$ is open in $\&, \otimes_{1}^{l}$. Since

$$
\pi(\mathbb{B} l)=\mathbb{B}_{1}^{l}-\pi\left(\mathscr{S}_{-}\right)
$$

by Lemma $6.3, \pi\left(\otimes^{l}\right)$ is closed in $\oiint_{1}^{l}$. Similarly $\pi\left(\&^{r}\right)$ is open and closed in (अ) ${ }_{1}^{r}$. Now

$$
\mathbb{B}_{1}=\pi\left(\mathscr{F}_{2}\right)=\pi\left(\mathscr{S}_{2}\right) \cup \pi\left(\mathscr{S}_{-}\right) \cup \pi\left(\mathscr{F}_{+}\right)
$$

and (as noted above) the latter sets are disjoint and also open by Lemma 6.6. But $\pi(\mathbb{B})=\pi\left(\mathscr{F}_{0}\right)$ by Lemma 6.2. Thus $\pi(\&)$ is open and closed in $\mathbb{B}_{1}$ and the proof of $(3)$ is complete.

Only (4) remains to be shown. Either $\pi(B)=\otimes_{1}$ or $\pi(\&)$ is properly contained in $\$_{1}$. Suppose that the latter holds. By Lemma 6.2, $\pi\left(\delta_{2}\right)=\pi(\&)$. But $\pi\left(\mathscr{F}_{2}\right)=\mathbb{B}_{1}$. Thus $\mathscr{F}_{2} \neq \mathscr{F}_{2}$ and the function $f$ defined on $\mathscr{F}_{2}$ (and on $\pi\left(\mathscr{F}_{2}\right)$ ) is not identically zero. Since $f$ is integral valued there is an integer $m>0$ and $T \in \mathscr{H}_{2}$ such that $|f(T)|=m$ and $m$ is minimal with respect to this property. By Corollary 5.5, $f$ is a homomorphism of $\pi\left(f_{2}\right)=\mathbb{B}_{1}$ into the additive group $J$ of integers. If we define $f_{1}$ on $\mathbb{S}_{1}$ by the rule $f_{1}=m^{-1} f$ then $f_{1}$ is a homomorphism 
of $\mathbb{B}_{1}$ onto $J$. The kernel of this homomorphism is $\pi\left(\mathscr{S}_{0}\right)=\pi(\mathbb{B})$ (Lemma 6.2). If $J$ is given the discrete topology then $f_{1}$ is an open mapping. Since the kernel is open in $\mathscr{F}_{1}$ by $(3)$, the inverse image under $f_{1}$ of any subset of $J$ is open in $\mathbb{B}_{1}$. Hence, $[11, \mathrm{p} .64], \mathbb{B}_{1} / \pi(\mathbb{B})$ is isomorphic, as a topological group, to $J$. This completes the proof of Theorem 6.1.

6.7. THEOREM. The following statements are equivalent:

(1) $X$ is not stable-like;

(2) $\mathscr{F}_{2}=\mathscr{F}_{0}$;

(3) $\pi(\mathbb{B})=\otimes_{1}$.

Proof. The equivalence of $(1)$ and (2) is given by Theorem 5.9. In the course of the proof of Theorem 6.1 it was shown that if $\pi(\&) \neq \mathbb{B}_{1}$ then $\delta_{2} \neq \mathcal{F}_{2}$ so that $(2)$ implies $(3)$. If $\pi(\&)=\mathbb{B}_{1}$ then, by Lemma $6.2, \pi\left(\tilde{S}_{0}\right)=\pi\left(\mathscr{S}_{2}\right)$. This shows that any element $T$ of $\tilde{F}_{\mathcal{L}}$ differs from an element of $\tilde{F}_{2}$ by a completely continuous transformation in $\mathbb{B}(X)$. Therefore, from Lemma 2.4, $\sqrt{2}=\sqrt{2} 0$.

6.8. Definition. We say that $\mathscr{X}$ is projection-stable if there exists an isomorphism in $\mathscr{E}(X)$ of $X$ onto a proper closed linear manifold $\Re$ where there is a ( continuous ) projection of $X$ on $\Re$.

Clearly if $\chi$ is stable-like then $\chi$ is projection-stable. Whether or not the converse is true is an open question. The notion just defined is connected with the notions of Theorem 6.1 by the following result.

6.9. THEOREM. The following statements are equivalent:

(1) $¥$ is not projection-stable;

(2) $\otimes^{l}=B^{r}=\&$;

(3) $\pi(B)=B_{1}$ and $\otimes_{1}=\&_{1}^{l}=B_{1}^{r}$.

Proof. If $X$ is not projection-stable then, by [14, Theorem 3.15], अ8 $C$ \& \& so

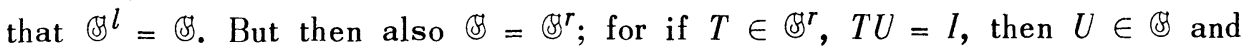
$T=U^{-1} \in$ अ). Thus (1) implies (2). Assume (2). By Theorem 6.1 we see that

$$
\pi(\&)=\pi\left(\&^{l} \cup \otimes^{r}\right)=\&_{1}^{l} \cup \oiint_{1}^{r} .
$$

But $\pi(\&) \subset \otimes_{1}$. Hence

$$
\mathbb{B}_{1}^{l}=\mathbb{B}_{1}^{r}=\otimes_{1} \text { and } \pi(\mathbb{B})=\mathbb{B}_{1} \text {. }
$$

Assume (3). If $\mathfrak{X}$ were projection-stable then by [14, Theorem 3.15] there 
exists $T \in \mathbb{B}^{l}, T \notin \&$. But $\pi(T) \in \mathscr{S}_{1}^{l}=\oiint_{1}$. Hence $T \in \mathcal{S}_{2}$. By its nature $f(T)>0$. However, from Theorem $6.7, \sqrt[5]{2}=\sqrt{2}$, which is a contradiction.

\section{REFERENCES}

1. F. V. Atkinson, The normal solubility of linear equations in normed spaces, Mat. Sbornik 28 (1951), 3 - 14. (Russian).

2. S. Banach, Théorie des opérations linéaires, Monografje Matematyczna, T.I. Warsaw, 1932.

3. J. W. Calkin, Two-sided ideals and congruences in the ring of bounded operators in Hilbert space, Ann. of Math. 42 (1941), 839-873.

4. J. Dieudonné, Sur les homomorphisms d'espaces normés, Bull. Sci. Math. (2) 67 (1943), $72-84$.

5. N. Dunford and B. J. Pettis, Linear operations on summable functions, Trans. Amer. Math. Soc. 47 ( 1940$), 323$ - 392.

6. I. C. Gohberg, On linear equations in normed spaces, Doklady Akad. Nauk SSSR (N.S. ) 76 (1951), 477-480. (Russian).

7. E. Hille, Functional analysis and semi-groups, Amer. Math. Soc. Colloquium Publications, vol. 31, New York, 1948.

8. I. Kaplansky, Topological rings, Amer. J. Math. 69 (1947), 153 - 183.

9. C. C. MacDuffee, The theory of matrices, Chelsea Publishing Company, New York, 1946.

10. G. W. Mackey, On infinite-dimensional linear spaces, Trans. Amer. Math. Soc. 57 (1945), 155 - 207.

11. L. Pontrjagin, Topological groups, Princeton University Press, 1939.

12. C. E. Rickart, The singular elements of a Banach algebra, Duke Math. J. 14 (1947), $1063-1077$.

13. J. Schauder, Über lineare vollstetige Funktionaloperationen, Studia Math. 2 (1930), $183-196$.

14. B. Yood, Transformations between Banach spaces in the uniform topology, Ann. of Math. 50 (1949), 486-503.

15. - Properties of linear transformations invariant under addition of a completely continuous transformation, Duke Math. J. 18 (1951), 599-612.

16. K. Yosida and S. Kakutani, Operator-theoretic treatment of Markoff's process and mean ergodic theorem, Ann. of Math., 42 (1941), 188-228.

\section{UNIVERSITY OF OREGON}




\section{PACIFIC JOURNAL OF MATHEMATICS}

\section{EDITORS}

\author{
M.M. SCHIFFER * \\ Stanford University \\ Stanford, California \\ E. HEWITT \\ University of Washington \\ Seattle 5, Washington
}

\section{R.P. DILWORTH}

California Institute of Technology Pasadena 4, California

\section{E. F. BECKENBACH**}

University of California

Los Angeles 24, California

\section{ASSOCIATE EDITORS}

$\begin{array}{llll}\text { H. BUSEMANN } & \text { P.R. HALMOS } & \text { BØRGE JESSEN } & \text { J. J. STOKER } \\ \text { HERBERT FEDERER } & \text { HEINZ HOPF } & \text { PAUL LÉVY } & \text { E.G. STRAUS } \\ \text { MARSHALL HALL } & \text { R.D. JAMES } & \text { GEORGE PÓLYA } & \text { KÔSAKU YOSIDA }\end{array}$

\section{SPONSORS}

UNIVERSITY OF BRITISH COLUMBIA

CALIFORNIA INSTITUTE OF TECHNOLOGY

UNIVERSITY OF CALIFORNIA, BERKELEY

UNIVERSITY OF CALIFORNIA, DAVIS

UNIVERSITY OF CALIFORNIA, LOS ANGELES

UNIVERSITY OF CALIFORNIA, SANTA BARBARA

UNIVERSITY OF NEVADA

OREGON STATE COLLEGE

UNIVERSITY OF OREGON
UNIVERSITY OF SOUTHERN CALIFORNIA STANFORD RESEARCH INSTITUTE STANFORD UNIVERSITY WASHINGTON STATE COLLEGE UNIVERSITY OF WASHINGTON

AMERICAN MATHEMATICAL SOCIETY HUGHES AIRCRAFT COMPANY

Mathematical papers intended for publication in the Pacific Journal of Mathematics should be typewritten (double spaced), and the author should keep a complete copy. Manuscripts may be sent to any of the editors. Manuscripts intended for the outgoing editors should be sent to their successors. All other communications to the editors should be addressed to the managing editor, E.G. Straus, at the University of California Los Angeles 24, California.

50 reprints of each article are furnished free of charge; additional copies may be obtained at cost in multiples of 50 .

The Pacific Journal of Mathematics is published quarterly, in March, June, September, and December. The price per volume (4 numbers) is $\$ 12.00$; single issues, $\$ 3.50$; back numbers (Volumes $1,2,3$ ) are available at $\$ 2.50$ per copy. Special price to individual faculty members of supporting institutions and to individual members of the American Mathematical Society: $\$ 4.00$ per volume; single issues, $\$ 1.25$.

Subscriptions, orders for back numbers, and changes of address should be sent to the publishers, University of California Press, ,Berkeley 4, California.

Printed at Ann Arbor, Michigan. Entered as second class matter at the Post Office, Berkeley, California.

* To be succeeded in 1955, by H.L. Royden, Stanford University, Stanford, California.

** To be succeeded in 1955, by E.G. Straus, University of California, Los Angeles 24, Calif.

UNIVERSITY OF CALIFORNIA PRESS - BERKELEY AND LOS ANGELES 


\section{Pacific Journal of Mathematics}

\section{Vol. 4, No. $4 \quad$ August, 1954}

Paul Civin, Orthonormal cyclic groups .................... 481

Kenneth Lloyd Cooke, The rate of increase of real continuous solutions of

algebraic differential-difference equations of the first order ......... 483

Philip J. Davis, Linear functional equations and interpolation series . . . . . 503

F. Herzog and G. Piranian, Sets of radial continuity of analytic functions . . 533

P. C. Rosenbloom, Comments on the preceding paper by Herzog and

Piranian ............................................. 539

Donald G. Higman, Remarks on splitting extensions .............. 545

Margaret Jackson, Transformations of series of the type ${ }_{3} \Psi_{3} \ldots \ldots \ldots \ldots 557$

Herman Rubin and Patrick Colonel Suppes, Transformations of systems of relativistic particle mechanics ....................... 563

A. Seidenberg, On the dimension theory of rings. II .............. 603

Bertram Yood, Difference algebras of linear transformations on a Banach

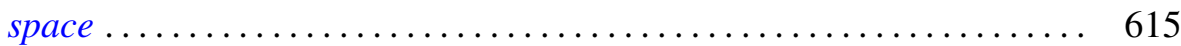

